\title{
Role of cathepsin $B$ in intracellular trypsinogen activation and the onset of acute pancreatitis
}

\author{
Walter Halangk, ${ }^{1}$ Markus M. Lerch, ${ }^{2}$ Barbara Brandt-Nedelev, ${ }^{1}$ Wera Roth, ${ }^{3}$ \\ Manuel Ruthenbuerger, ${ }^{2}$ Thomas Reinheckel, ${ }^{1}$ Wolfram Domschke, ${ }^{2}$ Hans Lippert, ${ }^{1}$ \\ Christoph Peters, ${ }^{3}$ and Jan Deussing ${ }^{3}$
}

${ }^{1}$ Department of Surgery, Division of Experimental Surgery, Otto-von-Guericke-Universität, Magdeburg, Germany
${ }^{2}$ Department of Medicine B, Westfälische Wilhelms-Universität, Münster, Germany
${ }^{3}$ Department of Medicine I, Division of Medical Molecular Biology, Albert-Ludwigs-Universität, Freiburg, Germany

Address correspondence to: Markus M. Lerch, Department of Medicine B, Westfälische Wilhelms-Universität, Albert-Schweitzer-Strasse 33, 48129 Münster, Germany.

Phone: 49-251-83-47559; Fax: 49-251-83-49504; E-mail: markus.lerch@uni-muenster.de.

Walter Halangk and Markus M. Lerch contributed equally to this work.

Received for publication January 18, 2000, and accepted in revised form July 25, 2000.

\begin{abstract}
Autodigestion of the pancreas by its own prematurely activated digestive proteases is thought to be an important event in the onset of acute pancreatitis. The mechanism responsible for the intrapancreatic activation of digestive zymogens is unknown, but a recent hypothesis predicts that a redistribution of lysosomal cathepsin B (CTSB) into a zymogen-containing subcellular compartment triggers this event. To test this hypothesis, we used CTSB-deficient mice in which the ctsb gene had been deleted by targeted disruption. After induction of experimental secretagogue-induced pancreatitis, the trypsin activity in the pancreas of $c t s b^{-/-}$animals was more than $80 \%$ lower than in $c t s b^{+/+}$animals. Pancreatic damage as indicated by serum activities of amylase and lipase, or by the extent of acinar tissue necrosis, was $50 \%$ lower in $c t s b^{-/}$animals. These experiments provide the first conclusive evidence to our knowledge that cathepsin B plays a role in intrapancreatic trypsinogen activation and the onset of acute pancreatitis.
\end{abstract}

J. Clin. Invest. 106:773-781 (2000).

\section{Introduction}

Acute pancreatitis has long been considered to be an autodigestive disorder, in which the parenchymal tissue of the organ is destroyed by its own digestive proteases (1). Under physiological conditions, pancreatic proteases are synthesized as inactive precursor zymogens and stored by the acinar cells in zymogen granules. Autodigestion of the gland therefore requires premature activation of these zymogens. How and where such a premature and intrapancreatic activation of digestive proenzymes is initiated in the course of pancreatitis has been the subject of several investigations (2). Recent studies strongly suggest that the early pathophysiological events that eventually lead to necrosis of pancreatic tissue originate in the acinar cell $(3,4)$ and involve the intracellular presence of active trypsin $(4,5)$, a serine proteinase capable of activating other pancreatic zymogens. In pancreatic acinar cells, cytoplasmic vacuoles have been identified as the subcellular compartment in which premature trypsinogen activation begins within minutes after induction of experimental pancreatitis (6, 7). The molecular mechanisms responsible for the intracellular activation of trypsinogen, however, have remained elusive. One hypothesis predicts that the lysosomal cysteine proteinase cathepsin B (CTSB) plays an essential role in this process (8). The largely circumstantial evidence for this "cathepsin B hypothesis" is based on the following observations: (a) CTSB was shown to activate trypsinogen in vitro (9); (b) during the initial phase of acute pancreatitis in several animal models, a redistribution of CTSB into a zymogen granule-containing subcellular compartment was detected by density-gradient centrifugation (10); (c) in the same pancreatitis models, lysosomal enzymes were detected by immunogold electron microscopy in secretory organelles that also contained digestive enzymes, e.g., trypsinogen (11). Experimental approaches to show an essential role of CTSB in premature zymogen activation by inhibition of this lysosomal enzyme with synthetic inhibitors rendered contradictory results either increasing (12) or decreasing (13) premature zymogen activation, or failing to improve the course of experimental pancreatitis (14). To test the cathepsin B hypothesis more directly and to overcome the shortcomings of lysosomal enzyme inhibitors, which have only limited specificity for CTSB, we used a CTSB-deficient mouse strain that was generated by targeted disruption of the cts $b$ gene (15). CTSB-deficient mice were found to be viable and fertile, and they did not show any spontaneous pathological phenotype. Experimental pancreatitis was induced in these animals and their wild-type controls by injection of supramaximal concentrations of the 
secretagogue caerulein. When the course of pancreatitis in both groups of animals was compared, we found that the premature and intracellular activation of trypsinogen that is followed by acinar cell necrosis largely depends on the presence of CTSB.

\section{Methods}

Construction of a ctsb gene targeting construct and generation of CTSB-deficient mice. The CTSB-deficient mice used in this study were previously reported and used for another purpose (15) but without the details of their construction that is provided here. A $14.6-\mathrm{kb}$ genomic DNA fragment covering exons $2-8$ of the murine ctsb gene was isolated from a $129 / \mathrm{SvJ} \lambda$-FIX II-teratocarcinoma library (Stratagene, La Jolla, California, USA) by hybridization with a 440-bp exon 6to 7 -specific genomic DNA probe (16) generated by PCR. The phage clone was characterized by restriction mapping, and exon/intron boundaries were determined by DNA sequencing. A 7.7-kb HindIII-EcoRV DNA fragment covering exons 2-6 was subcloned into plasmid vector pBluescriptII SK(+) (Stratagene). The neomycin resistance cassette of PMC1 neopA (17) was inserted into a BglII site in exon 4 of this ctsb gene subclone utilizing BamHI-linker sites flanking the cassette. The insertion of the neomycin cassette introduces a premature stop codon into the open reading frame of the $c t s b$ gene. The resulting plasmid pMCB11/1neo (Figure 1a) was linearized with HindIII and introduced into E-14-1 cells (18) by electroporation. Embryonic stem (ES) cells were cultured as described by Kühn et al. (19). G418-resistant ES cell colonies were screened for homologous recombination by Southern analysis of genomic DNA digested with BglII and hybridized with probe B (Figure $1 \mathrm{~b}$ ). Targeted ES cell clones were confirmed by digestion of genomic DNA with KpnI and EagI and subsequent hybridization with probe A (Figure $1 \mathrm{~b}$ ). Targeted ES cell clones were microinjected into blastocysts of C57BL/6J mice as described by Köster et al. (20), and resulting male chimeras were mated to $\mathrm{C} 57 \mathrm{BL} / 6 \mathrm{~J}$ females. Mice were genotyped for the $c t s b$ gene mutation by Southern analysis of BglII digested genomic tail DNA using probe B. Heterozygous offspring were intercrossed for generation of $c t s b^{-} / c t s b^{-}$mice (Figure 1c). Mice used as wild-type controls in this study had the same outbred C57BL/6J × 129/SvJ genetic background and were kindly provided by P. Saftig (University of Göttingen, Göttingen, Germany). Animals used in this study were maintained and bred according to our institutional guidelines and animal facility protocols. All subsequent experiments including the induction of acute pancreatitis were performed in accordance with the same guidelines and after prior approval by the institutional animal care committee.

Northern and Western blot analysis. Total RNA of kidney from 8-week-old mice was prepared as described previously (21). Total RNA ( $8 \mu \mathrm{g})$ was separated in a formaldehyde agarose gel and processed as reported previously (22). Filters were hybridized with a 980-bp partial ctsb cDNA generated by RT-PCR (16) and a 280-bp cDNA fragment from murine glyceraldehyde3-phosphate dehydrogenase (G3PD) (23).

Lysosomes were enriched from kidney homogenates (24), and soluble lysosomal protein $(250 \mu \mathrm{g})$ was separated by $10 \%$ SDS-PAGE, blotted, and probed with a rabbit polyclonal antiserum raised against human CTSB (cross-reacting with murine CTSB; kindly provided by E. Weber (Halle, Germany) using an electrochemiluminescence (ECL) immunodetection system (Amersham, Freiburg, Germany) (25).

Detection of CTSB enzyme activity. CTSB proteolytic activity was determined in liver lysosomal fractions (24) by using the fluorogenic substrate Z-Arg-Arg-4methyl-coumarin-7-amide $(20 \mu \mathrm{M}$; Bachem California, Torrance, California, USA) as described by Barrett and Kirschke (26). Assays were performed in $50 \mathrm{mM}$ phosphate buffer ( $\mathrm{pH}$ 6.0) containing $2.5 \mathrm{mM}$ EDTA and $2.5 \mathrm{mM}$ dithiothreitol. Reaction mixtures were preincubated for 30 minutes at $37^{\circ} \mathrm{C}$ and incubated in the presence of substrate for 10 minutes. Incubations were stopped by addition of $100 \mathrm{mM}$ monochloroacetate ( $\mathrm{pH} 4.3$ ), and the release of 7-amino-4-methylcoumarin was monitored by spectrofluorometry. One unit corresponds to the enzyme activity liberating 1 $\mu \mathrm{mol}$ 7-amino-4-methylcoumarin per min.

Induction of experimental pancreatitis. Pancreatitis was induced in 6- to 8-week-old $\mathrm{CTSB}^{-/-}$and $\mathrm{CTSB}^{+/+}$mice weighing 20-24 g. After fasting for 18 hours with access to water ad libitum, the secretagogue caerulein (Pharmacia Biotech AB, Uppsala, Sweden) was administered in seven intraperitoneal injections of $50 \mu \mathrm{g} / \mathrm{kg}$ body weight at hourly intervals (27). Saline-injected animals served as controls.

Preparation of serum and tissue samples. Mice were sacrificed at intervals between 1 and 24 hours after the first intraperitoneal injection of caerulein. Whole blood samples were centrifuged at $4^{\circ} \mathrm{C}$, and serum was stored at $-80^{\circ} \mathrm{C}$ for further studies. Tissue from pancreas and lungs was removed on ice, weighed, immediately frozen in liquid nitrogen, and stored at $-80^{\circ} \mathrm{C}$. Tissue for the measurement of pancreatic enzyme activities was thawed and homogenized in iced medium containing $5 \mathrm{mM}$ MOPS, $1 \mathrm{mM} \mathrm{MgSO}_{4}$, and 250 $\mathrm{mM}$ sucrose ( $\mathrm{pH}$ 6.5). Samples were sonicated and centrifuged for 5 minutes at $16,000 \mathrm{~g}$. Sample preparation for trypsinogen activation peptide (TAP) assay was performed as described previously (28), supplementing the tissue homogenate with $1 \mathrm{mM}$ EDTA and $0.1 \%$ Triton X-100 and boiling the samples for 10 minutes. Supernatants generated at $16,000 \mathrm{~g}$ for 5 minutes were used in the subsequent assays. For MPO measurements (29), pancreatic or lung tissue was homogenized in $20 \mathrm{mM}$ potassium phosphate buffer $(\mathrm{pH} 7.4)$ and centrifuged for 10 minute at $10,000 \mathrm{~g}$. The pellet was resuspended in $50 \mathrm{mM}$ potassium phosphate buffer ( $\mathrm{pH}$ 6.0) containing $0.5 \%$ cetyltrimethylammonium bromide. The suspension was freeze-thawed four 
times, sonicated twice for 10 seconds each, and centrifuged at $10,000 \mathrm{~g}$ for 5 minutes.

To study whether the CCK-receptor signaling pathway had remained intact and the pancreas of CTSBdeficient mice responded to caerulein stimulation in the same manner as that of wild-type animals, we freshly prepared isolated pancreatic acini by collagenase digestion as reported previously (30) and incubated them in HEPES-buffered Krebs-Ringer solution ( $\mathrm{pH}$ 7.4) with various concentrations of caerulein. Amylase secretion over the course of 30 minutes was measured as amylase activity and expressed as percent of total amylase content of the acini.

Biochemical assays. Trypsin was measured at $37^{\circ} \mathrm{C}$ using $64 \mu \mathrm{M}$ BOC-Gln-Ala-Arg-7-amino-4-methylcoumarin (Bachem California) as a substrate (28). To increase specificity, trypsin activity was expressed as fluorescence increase sensitive to soybean trypsin inhibitor (SBTI; $2 \mu \mathrm{M}$ ). Trypsinogen content was measured as trypsin activity after preincubation with an excess amount of enteropeptidase over a period of 30 minutes. The trypsin activity was corrected for substrate cleavage by enteropeptidase. Tissue contents of trypsin and trypsinogen were standardized to a purified trypsin preparation (T-8003; Sigma Chemical Co., St. Louis, Missouri, USA) whose activity was determined by active site titration. Parallel titrations of the standard trypsin and mouse trypsin activity with SBTI showed that the specific activities of both were comparable. Amylase and lipase activities were determined by commercially available assays (Boehringer, Mannheim, Germany), and MPO activity was assayed after mixing $200 \mu \mathrm{L}$ of supernatant in $1.0 \mathrm{~mL}$ of 50 $\mathrm{mM}$ potassium phosphate buffer $(\mathrm{pH} 6.0)$ containing $0.53 \mathrm{mM} \mathrm{O}$-dianisidine and $0.15 \mathrm{mM} \mathrm{H}_{2} \mathrm{O}_{2}$. The initial increase in absorbance at $460 \mathrm{~nm}$ was measured at $30^{\circ} \mathrm{C}$. The results are expressed in units of MPO on the basis of 1 unit to oxidize $1 \mu \mathrm{mol} \mathrm{H}_{2} \mathrm{O}_{2}$ per minute. TAP was assayed using an enzyme immunoassay (Biotrin, Dublin, Ireland). IL-6 serum levels were measured by ELISA (Biosource International, Camarillo, California, USA) and protein concentrations were determined according to Bradford (31).

Morphology and morphometry. At selected time intervals of pancreatitis, tissue samples were collected from both lungs as well as the head, body, and tail of the pancreas of $\mathrm{CTSB}^{+/+}$and $\mathrm{CTSB}^{-/-}$mice, immediately immersed in iced fixative, and processed for electron microscopy, paraffin histology, or cryosections. For experiments involving the detection of apoptotic cells strips of pancreas were fixed in iced $125 \mathrm{mM}$ phosphate buffer ( $\mathrm{pH}$ 7.4) containing 4\% formaldehyde for 90 minutes and, after $5-\mu \mathrm{m}$ cryosections were recovered on coverslips, postfixed with iced acetone for 30 seconds. Free 3'OH-DNA termini were labeled using the terminal deoxynucleotidyl transferase (TdT) method with fluoresceinlabeled digoxigenin nucleotides as reported previously (32). Fluorescent micrographs were taken at random at 40× (Nikon Diaphot 200; excitation 485 $\mathrm{nm}$, emission $530 \mathrm{~nm}$; Nikon, Düsseldorf, Germany). To quantitate nuclei containing apoptotic bodies in comparison to all nuclei, sections were double labeled with the DNA dye 4,6-diamidino-2-phenylindole (DAPI; excitation $335 \mathrm{~nm}$, emission $450 \mathrm{~nm}$ ). Prints were coded for morphometry, and the number of nuclei containing fluorescein-positive apoptotic bodies were counted in a blinded fashion, calculated as percent of all DAPI-positive nuclei and expressed as percent of cells \pm SEM. From three or more experiments in each group, approximately 500 printed fields, which contained an average of 125 cells per field were evaluated in this manner.

For resin-embedded thin sections, strips of pancreas measuring $1.0 \times 0.5 \mathrm{~mm}$ were immediately fixed in 125 $\mathrm{mM}$ phosphate buffer ( $\mathrm{pH}$ 7.4) containing $2 \%$ glutaraldehyde/ $2 \%$ formaldehyde for 90 minutes, rinsed extensively in the same buffer, and post-fixed in $2 \%$ $\mathrm{OsO}_{4}$. Tissue blocks were dehydrated in ethanol and embedded in Epon 812. Semithin sections were stained with methylene blue and examined by light microscopy. Selected areas, chosen for detailed study, were thin-sectioned using a LKB Ultratome (LKB, Bromma, Sweden), picked up on uncoated copper grids, double stained with uranyl acetate and lead citrate, and examined on a Philips EM10 transmission electron microscope (Philips, Eindhoven, The Netherlands). To quantitate the ultrastructural changes, cross sections of entire acini containing a minimum of ten cells including the nucleus and plasma membrane were randomly chosen, photographed, and printed on photographic paper at a calibrated magnification of 1,500. Prints of each section were coded, and the number of cytoplasmic vacuoles and cells undergoing necrosis (resolution of organelle-confining or cell-limiting plasma membranes) was morphometrically evaluated as already described here and expressed per total number of cells $(3,33)$. A minimum of 500 cells per experiment was evaluated in this manner.

Data presentation and statistical analysis. Data in graphs are expressed as means \pm SEM. Statistical comparison between the $\mathrm{CTSB}^{+/+}$and the $\mathrm{CTSB}^{-/-}$group at various time intervals was done by Student's $t$ test for independent samples using SPSS for Windows (SPSS Inc., Chicago, Illinois, USA). Differences were considered significant at a level of $P<0.05$. Data presentation was performed with ORIGIN for Windows (Microcal Software, Northampton, Massachusetts, USA).

\section{Results}

Targeted disruption of the ctsb gene and generation of CTSBdeficient mice. The strategy for generating CTSB-deficient mice is summarized in Figure $1, a-c$, and its respective legend. To test for inactivation of the ctsb gene in homozygous mutant mice, its expression was studied by Northern and Western blot analyses. Neither ctsb transcript was detectable in RNA from homozygous mutant mice (Figure 1d) nor was CTSB 
a
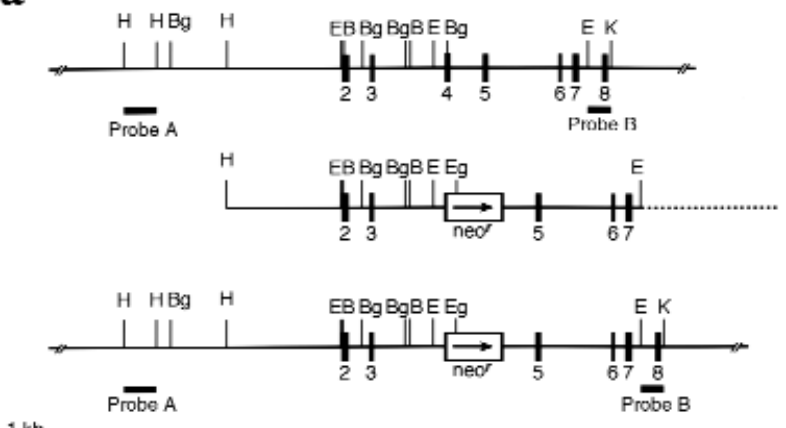

b

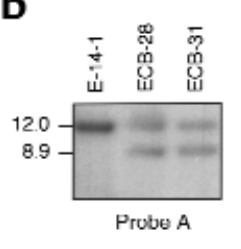

d

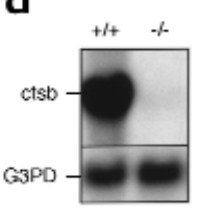

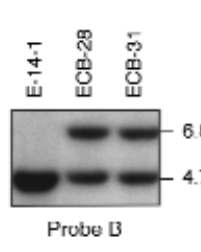

e

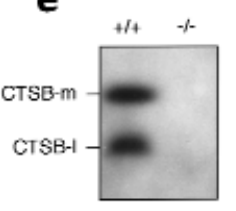

C

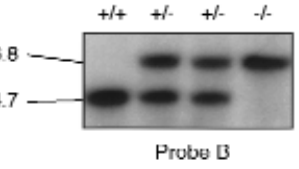

f

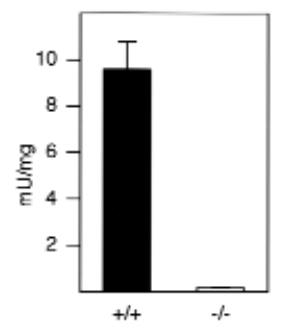

\section{Figure 1}

Targeted disruption of the $c t s b$ gene. (a) Strategy for inactivation of the ctsb-gene by homologous recombination in embryonic stem cells. (Top) Genomic structure and partial restriction map of the wild-type ctsb-locus $(15,47,48)$. Exons are numbered and indicated by black boxes. External probes A and B used for Southern blot analyses are represented by black bars. (Middle) Targeting construct PMCB-11/1neo with $7.7 \mathrm{~kb}$ homology to the ctsb gene locus. A neomycin-resistance cassette was inserted into a Bglll site in exon 4. The arrow marks the direction of transcription of the neomycin gene. (Bottom) Predicted ctsb-locus after homologous recombination. Broken line: plasmid vector pBluescriptII SK (+). B, BamHI; Bg, BgIII; E, EcoRV; Eg, Eagl; H, HindIII; K, KpnI. (b) Southern blot analysis of targeted ES cell clones. The 3 '-external probe B was hybridized to Bglll digested genomic DNA from wild-type E-141 ES cells and ES cell clones ECB-28 and ECB-31 with a targeted ctsb allele as indicated by the presence of an additional 6.8-kb DNA fragment. The 5 '-external probe A was hybridized to Kpnl and Eagl digested DNA from the same ES cell clones, confirming homologous recombination in clones ECB-28 and ECB-31. (c) Southern blot analysis of tail DNA from offspring of an intercross of ctsb heterozygotes. Genotypes of progeny are indicated. (d) Northern blot analysis of ctsb mRNA expression. Total RNA was hybridized to a partial ctsb cDNA and a murine glyceraldehyde-3-phosphate dehydrogenase probe (G3PD). (e) Western blot analysis of CTSB protein expression from kidney lysosomal extracts; CTSB-m: mature CTSB (single chain form); CTSB-I: large subunit (two chain form). (f) CTSB enzyme activity in liver lysosomal fractions expressed as $\mathrm{mU} / \mathrm{mg}$ protein $( \pm \mathrm{SD} ; n=3)$.

protein present in lysosomal protein extracts (Figure 1e). Using Z-Arg-Arg-4-methyl-coumarin-7-amide as substrate, no CTSB-activity was detected in liver of $c t s b^{-} / c t s b^{-}$mice (Figure 1f). These data indicate that the $c t s b$ gene was successfully inactivated.

Phenotypic analysis of $c t s b^{-} / c t s b^{-}$mice. Homozygous CTSB-deficient mice exhibited normal development at birth and showed no apparent neurological or behavioral deficits. Postnatal development was indistinguishable from that of heterozygous and wild-type littermates. Inspection of external and internal organs at different ages did not reveal any macroscopic abnormalities in $c t s b^{-} / c t s b^{-}$mice. On histopathological investigation of various organ systems including brain, heart, lung, liver, spleen, thymus, pancreas, stomach, intestine, ovary, kidney, skeletal muscle, and lymph nodes, no pathological alterations could be detected in CTSB-deficient mice (data not shown). Homozygous mutant mice were fertile and reproduced normally. FACS analysis of lymphoid tissues (thymus, spleen and lymph nodes) did not reveal any abnormalities of $\mathrm{T}$ or B lymphocytes (data not shown).

Isolated pancreatic acini that were prepared from the pancreas of CTSB-deficient and wild-type mice responded to stimulation with caerulein in parallel manner, i.e., with increasing rates of amylase secretion up to a caerulein concentrations of $10^{-11} \mathrm{M}$, and with decreasing rates of amylase secretion at supramaximal caerulein concentrations of up to $10^{-9} \mathrm{M}$ (Figure 2a). These results indicate that the CCKreceptor signaling pathway was not affected by the deletion of the ctsb gene and that the biphasic stimulus response to caerulein had remained intact in CTSB-deficient mice.

Intrapancreatic trypsinogen activation during experimental pancreatitis. Because the premature activation of trypsinogen as an initiating event of pancreatitis depends on the amount of trypsinogen that is available in the pancreas, the pancreatic trypsinogen content was determined in CTSB-deficient and control mice. The resting levels of pancreatic trypsinogen in $\mathrm{CTSB}^{-/-}$mice amounted to about twice those found in control mice (Figure 2b). During the course of experimental pancreatitis, the trypsinogen content rose in both groups of animals, which is due to a blockage of enzyme secretion (34) in the presence of ongoing protein synthesis. When trypsin activity rather than trypsinogen content was determined, only very little substrate cleavage was found in the pan- 


\section{Figure 2}

Trypsinogen activation during experimental pancreatitis. In the experiment shown in a, dispersed acini were generated from the pancreas of CTSB-deficient and wild-type animals as described in Methods and incubated in different concentrations of caerulein. Amylase secretion over the course of 30 minutes was expressed in percent of total amylase content. In $\mathbf{b}$-d, the pancreatitis time course is shown over a 24-hour period for (b) pancreatic trypsinogen content, (c) free trypsin activity in the pancreas, and (d) trypsinogen activation peptide (TAP) in the pancreas. Data points represent the means of four or more pancreatitis animals ( $n \geq 3$ for controls) at each interval \pm SEM. ASignificant differences $(P<0.05)$ between the $\mathrm{CTSB}^{+/+}$and the $\mathrm{CTSB}^{-/-}$mice. a

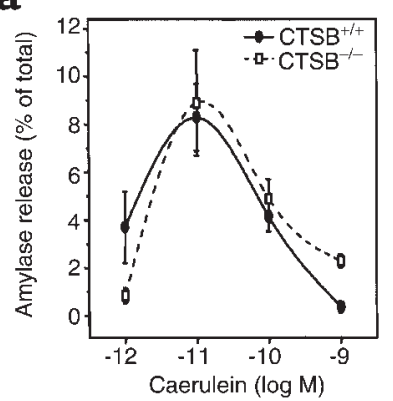

C

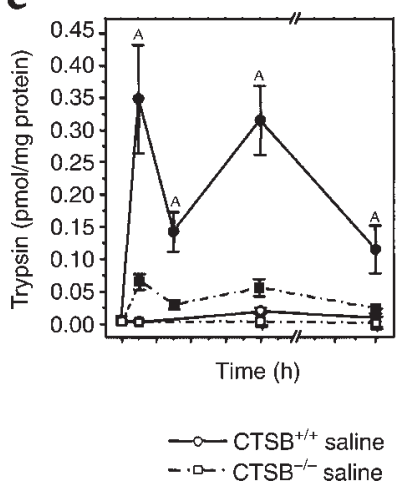

b

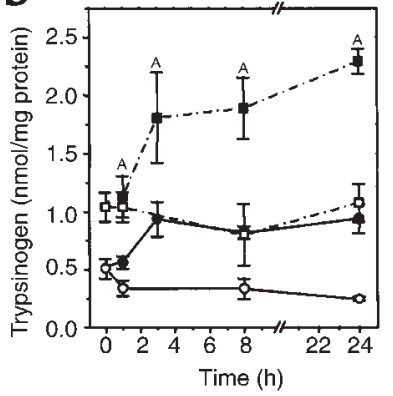

d

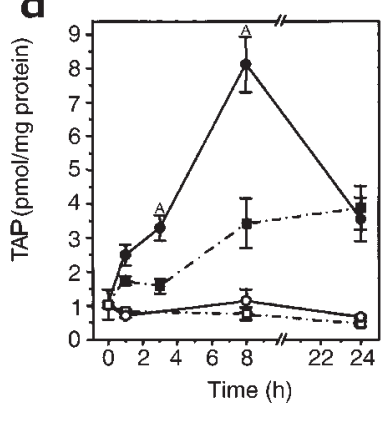

$\longrightarrow \mathrm{CTSB}^{+/+}$caerulein creas of untreated or saline-injected $\mathrm{CTSB}^{+/+}$or $\mathrm{CTSB}^{-/-}$mice (Figure 2c). The intraperitoneal injection of caerulein was followed by a significant activation of trypsinogen in the pancreas of $\mathrm{CTSB}^{+/+}$and in that of $\mathrm{CTSB}^{-/-}$mice as well. At all time intervals during the course of experimental pancreatitis, however, the pancreatic trypsin activity was significantly lower in $\mathrm{CTSB}^{-/}$mice and reached only $18-24 \%$ of the activity levels found in wild-type controls. When, instead of measuring trypsin activity, the presence of TAP, the $\mathrm{NH}_{2}$-terminal pentapeptide that is cleaved from trypsinogen during the conversion to active trypsin, was measured as an indicator of premature trypsinogen activation, a similar reduction in premature trypsinogen activation during the pancreatitis of $\mathrm{CTSB}^{-/}$mice was found (Figure 2d). The TAPimmunoreactivity levels of $\mathrm{CTSB}^{-/-}$mice reached only $38-50 \%$ of those found in the pancreas of $\mathrm{CTSB}^{+/+}$animals during the course of pancreatitis. Less than 1\% of the total trypsinogen content in the pancreas participated in this premature and intrapancreatic conversion to active trypsin.

\section{Pancreatic damage during experimental pancreatitis}

Serum pancreatic enzyme activities and interstitial edema. Elevated amylase and lipase activities appeared as early as 1 hour after the first caerulein injection in the serum of CTSB ${ }^{+/+}$as well as of CTSB-/- mice (Figure 3, a

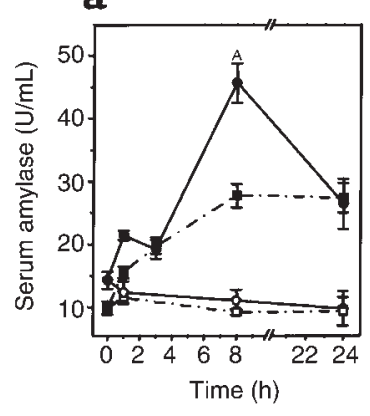

b

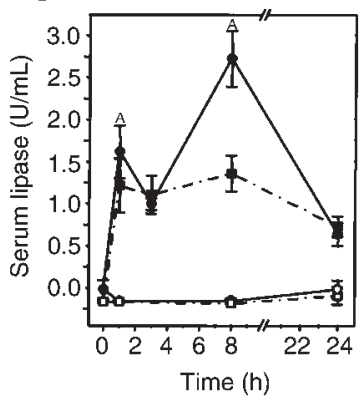

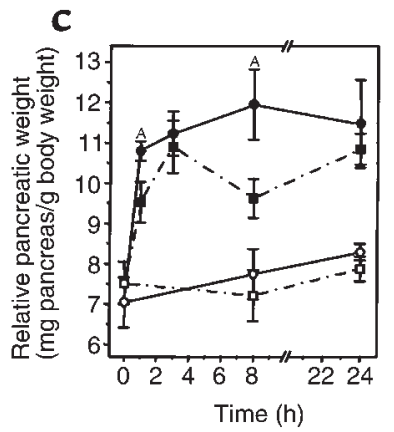

$\longrightarrow-\mathrm{CTSB}^{+/+}$saline
$-\bullet-\mathrm{CTSB}^{-/-}$saline

Figure 3

Pancreatic damage during experimental pancreatitis. The time course over 24 hours is shown for (a) serum amylase activity, (b) serum lipase activity, and (c) pancreatic edema. Enzyme activities were measured as indicated in Methods, and the increase in pancreatic water content was determined indirectly as increase in pancreatic weight. Data points represent the means of six or more pancreatitis animals $(n \geq 4$ for controls) at each interval \pm SEM. ASignificant differences $(P<0.05)$ between the $\mathrm{CTSB}^{+/+}$and the $\mathrm{CTSB}^{-/-}$mice. 
$\mathrm{a}$ and $\mathrm{b})$. One hour after the last intraperitoneal injection of caerulein, which in this model of pancreatitis represents the peak of hyperamylasemia and lipasemia, respectively, the serum amylase and lipase
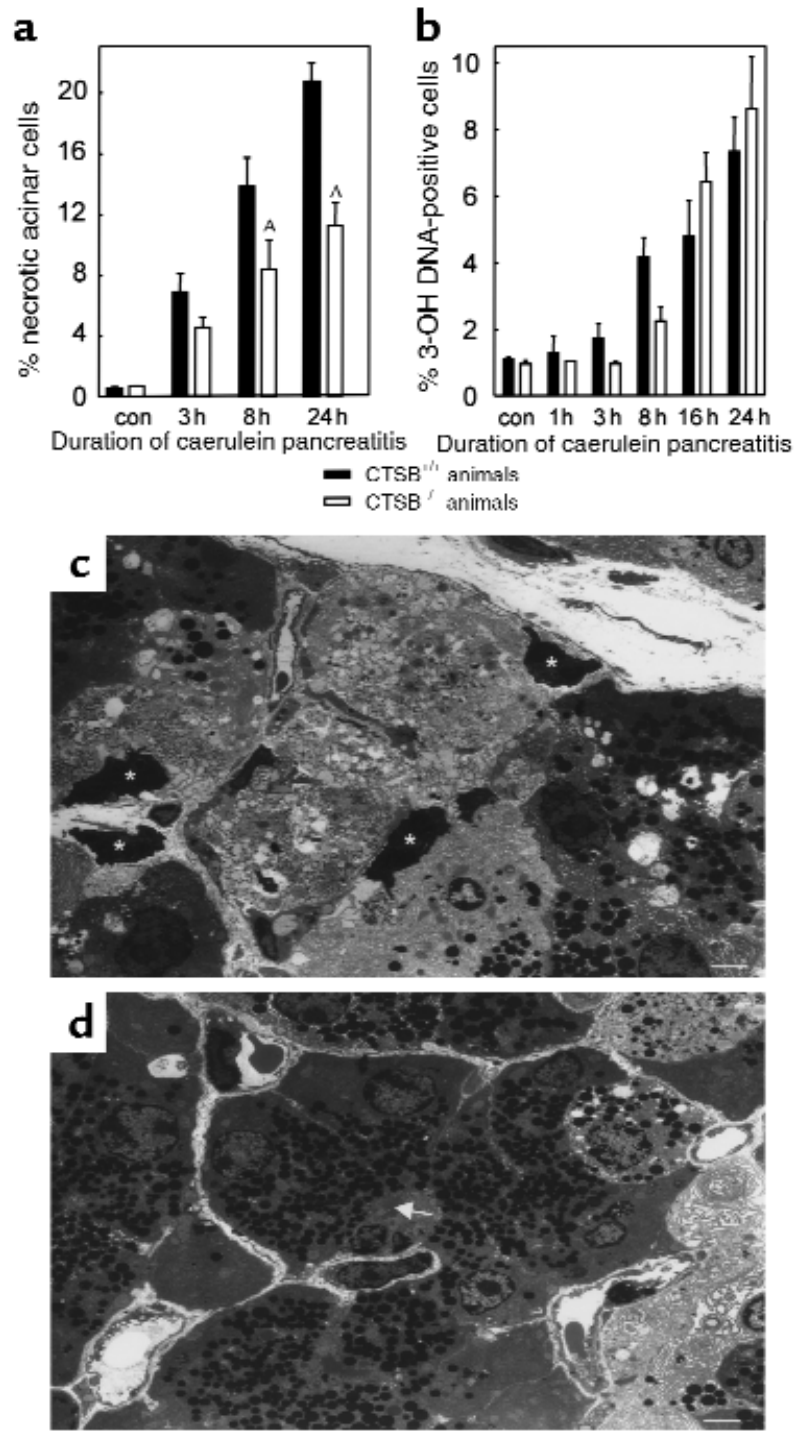

Figure 4

Pancreatic necrosis and apoptosis during experimental pancreatitis. The time course over 24 hours is shown for (a) the percentage of acinar cells that had undergone necrosis and (b) the percentage of cells that had undergone apoptosis after the induction of pancreatitis. The appearance of necrotic acinar cells was quantitated in electron microscopy sections and the number of apoptotic cells was determined using fluorescence labeling of DNAstrand brakes and was expressed as percentage of all DAPI-positive nuclei. Data points represent the means of three or more animals at each interval \pm SEM. ASignificant differences $(P<0.05)$ between the $\mathrm{CTSB}^{+/+}$and the $\mathrm{CTSB}^{-/-}$mice. In c, a representative micrograph is shown from the pancreas of a wild-type animal after 24 hours of pancreatitis. Note the prominent areas of acinar cell necrosis with resolution of cellular membranes and organelles as well as dark condensed nuclei (asterisks). In d, a corresponding section from a CTSB ${ }^{-/-}$animal is shown. Here more intact acinar cells with densely packed zymogen granules around the acinar lumen (arrow) are seen, and the area of necrosis is confined to the right-hand margin of the micrograph. Calibration bars $=10 \mu \mathrm{m}$. activities were $46 \%$ lower in $\mathrm{CTSB}^{-/-}$mice compared with their wild-type controls. The pancreatic content of either amylase or lipase was not reduced in the $\mathrm{CTSB}^{-/-}$animals (data not shown).

A further parameter of pancreatic damage in clinical as well as in experimental pancreatitis is the formation of intercellular fluid collections termed pancreatic edema. Although the development of pancreatic edema in mice is much less prominent than in the equivalent rat model of pancreatitis, and the differences between the increases in pancreatic water content of $\mathrm{CTSB}^{-/-}$ and $\mathrm{CTSB}^{+/+}$mice were not large, they reached significant levels at 1 hour and 8 hours of the evolution of acute pancreatitis (Figure 3c).

Pancreatic tissue necrosis and apoptosis. The course of clinical acute pancreatitis and that of various animal models of the disease can be complicated by significant necrosis of pancreatic tissue, and the extent of necrosis correlates with disease severity (35). In our study, the first ultrastructural change in acinar cells was a progressive dilatation of the endoplasmic reticulum and the formation of cytoplasmic vacuoles that either contained a homogeneous or a partially condensed protein content or, alternatively, fragments of organelles. Some of these vacuoles could thus clearly be identified as autophagosomes, whereas others may have derived from secretory vesicles. Although the number and size of these vacuoles increased over time, their occurrence was not nearly as frequent as in the equivalent rat model of secretagogue-induced pancreatitis (36). Neither the quality of these changes nor their frequency in affected cells differed in the course of pancreatitis of $\mathrm{CTSB}^{-/-}$and $\mathrm{CTSB}^{+/+}$animals (data not shown). The most severe morphological alteration observed during experimental pancreatitis was the necrosis of either individual acinar cells or the appearance of small tissue areas composed of necrotic acinar cells (Figure 4).

When the percentage of necrotic acinar cells was quantified in $\mathrm{CTSB}^{+/+}$and $\mathrm{CTSB}^{-/-}$mice, the amount of tissue necrosis was up to $48 \%$ lower in the latter (Figure 4a). The greatest extent of acinar tissue necrosis and the greatest difference between the $\mathrm{CTSB}^{+/+}$and the $\mathrm{CTSB}^{-/}$groups of mice were found after 24 hours of secretagogue-induced pancreatitis. Because cell death during the course of acute pancreatitis is known to occur not only as a consequence of autodigestion and necrosis but also owing to a greatly increased rate of acinar cell apoptosis $(37,38)$, and given that CTSB has been implicated in the development of apoptosis (39), we compared the rate of apoptosis in the pancreas of $\mathrm{CTSB}^{+/+}$and $\mathrm{CTSB}^{-/-}$mice. Based on the number of fluorescein-labeled DNA-strand brakes, approximately 9\% of all cells had undergone cell death by apoptosis at the end of the 24-hour observation period. However, no difference in the overall rate of apoptosis between $\mathrm{CTSB}^{+/+}$and $\mathrm{CTSB}^{-/-}$mice was found (Figure $4 \mathrm{~b}$ ).

Extrapancreatic damage during experimental pancreatitis. To study whether CTSB plays a role in determining the extrapancreatic severity of the disease process, 
we studied serum levels of IL-6, a proinflammatory cytokine that is known to increase during pancreatitis (40), the degree of leukocyte infiltration of the pancreas and lungs in terms of tissue levels of MPO, and the morphological changes in the lungs during the course of the disease (29). In both $\mathrm{CTSB}^{+/+}$and $\mathrm{CTSB}^{-/-}$mice, the induction of pancreatitis was associated with increased serum IL-6 concentrations (Figure 5a) and with increased tissue levels of MPO in the pancreas (Figure $5 \mathrm{~b}$ ) and lungs (Figure $5 \mathrm{c}$ ). The measurement of neither parameter resulted in statistically significant or biologically meaningful differences between the two groups, with the sole exception of serum IL- 6 measurements at 24 hours. Here, the declining IL-6 levels toward the end of the experiment were somewhat lower $(P=0.035)$ in the CTSB-deficient animals. The morphological changes in the pulmonary tissue consisted of alveolar fluid accumulation and a progressive thickening, hyperemia, and neutrophil infiltration of the interalveolar tissue. Neither of these alterations was found to differ between the lungs of $\mathrm{CTSB}^{+/+}$and $\mathrm{CTSB}^{-/-}$animals in the course of pancreatitis (Figure $5 \mathrm{~d}$ ).

\section{Discussion}

A century ago, when the digestive properties of pancreatic secretions became known, the morphological appearance of pancreatitis on autopsy sections suggested that the disease represents an autodigestive process (1). This view has not changed, and according to our current understanding, the onset of the disease involves a premature and intrapancreatic activation of proteolytic enzymes (2). Digestive proteases are, however, synthesized and secreted as inactive precursor zymogens, and the pancreatic acinar cell possesses a whole variety of protective mechanisms that either prevent premature zymogen activation or inhibit protease activity (41). The question, therefore, remains as to which molecular factors either trigger the initial protease activation or permit the cellular defenses to be overcome. One of the hypotheses that have attempted to address this issue predicts that CTSB, a lysosomal cysteine proteinase, is critically involved in the initial trypsinogen activation (8). This cathepsin B hypothesis is based on the observations that CTSB can activate trypsinogen in vitro (9), that CTSB is redistributed to a zymogen-granule-enriched subcellular compartment (10), and that lysosomal enzymes colocalize with digestive zymogens during the early course of experimental pancreatitis (11). Although this cathepsin-hypothesis appears attractive from a cell biologic point of view, a number of experimental observations appear to be incompatible with its assumptions: (a) a colocalization of cathepsins with digestive zymogens has not only been observed in the initial phase of acute pancreatitis, but also under physiological control conditions and in secretory vesicles that are destined for regulated secretion from healthy pancreatic acinar cells (42); (b) a

redistribution of CTSB into a zymogen-enriched subcellular compartment can be induced in vivo by experimental conditions that interfere with lysosomal sorting and are neither associated with nor followed by the development of acute pancreatitis (43); (c) the administration of potent lysosomal enzyme inhibitors in vivo does not prevent the onset of acute experimental pancreatitis (14); (d) in experiments that used lysosomal enzyme inhibitors in vitro, an increase in the rate of intracellular trypsinogen activation, as well as a decrease in the rate of intracellular trypsinogen activation, has been reported $(12,13)$, and even a protective role against premature zymogen activation has been considered for CTSB $(44,45)$. In view of the limited specificity and bioavailability of the existing inhibitors for lysosomal hydrolases, the only remaining option to address the cathepsinhypothesis conclusively was therefore to use CTSBdeficient animals and to study them in an experimental model of acute pancreatitis.

a
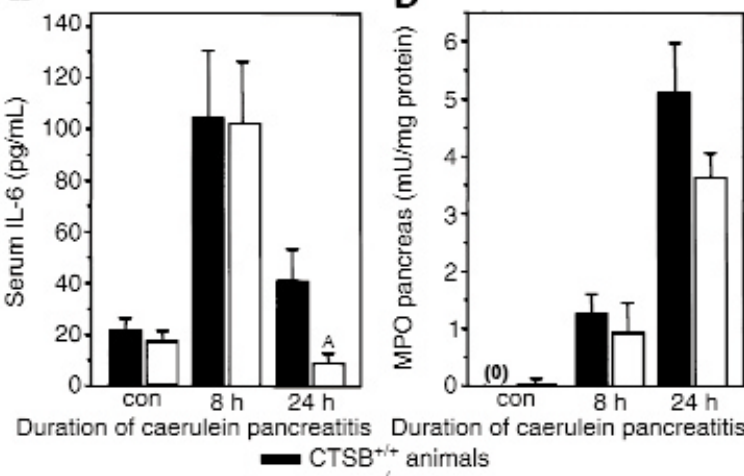
CTSB $^{+1+}$ animals
CTSB animals

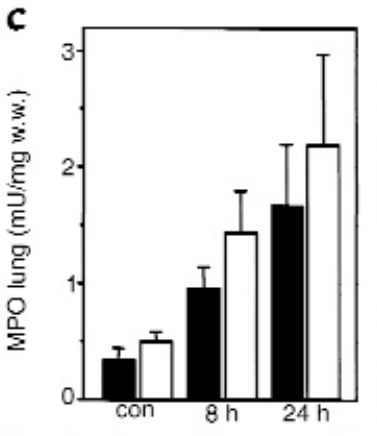

Duration of caerulein pancreatitis
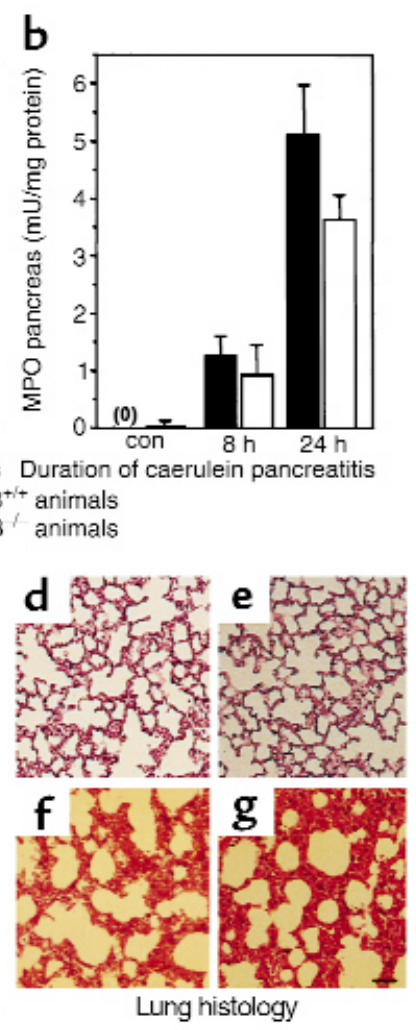

\section{Figure 5}

Extrapancreatic manifestations of experimental pancreatitis. The time course over 24 hours is shown for (a) serum concentrations of IL-6, (b) MPO levels in the pancreas, (c) MPO levels in the lungs, and (d-g) morphological changes in the lungs. Data points represent the means of five or more animals at each interval \pm SEM. ASignificant differences $(P<0.05)$ between $\mathrm{CTSB}^{+/+}$and $\mathrm{CTSB}^{-/-}$animals. $(\mathbf{d}-\mathbf{g})$ Representative examples for the lung histology of saline-injected control $\left(\mathrm{CTSB}^{+/+}, \mathbf{d} ; \mathrm{CTSB}^{-/-}, \mathbf{e}\right)$ mice, and pancreatitis animals at 24 hours of the disease $\left(\mathrm{CTSB}^{+/+}, \mathbf{f} ; \mathrm{CTSB}^{-/-}, \mathbf{g}\right)$ are shown. Note that the thickening of the interalveolar tissue, its hyperemia, and the infiltration by leukocytes are indistinguishable between the $\mathrm{CTSB}^{+/+}$and $\mathrm{CTSB}^{-/-}$group of animals. Calibration bar $=100 \mu \mathrm{m}$. 
We have chosen this approach and have used a strain of mice in which the ctsb gene was deleted by targeted disruption (15). In an extensive anatomical and functional characterization, we could show that the resulting $\mathrm{CTSB}^{-/-}$animals are phenotypically indistinguishable from their wild-type controls, that they reproduce normally, and that they carry no abnormalities in either their organ development or immune system. We used these CTSB-deficient animals to induce an experimental variety of acute pancreatitis. This secretagogue-induced model of the disease appears ideally suited to address the cathepsin-hypothesis for a number of reasons: (a) it is noninvasive and can be induced in mice and other small rodents (46); (b) it is associated with significant intrapancreatic trypsinogen activation that precedes acinar cell injury $(6,13)$; and (c) many of the local and systemic alterations known to be relevant for the human disease are found in this animal model including hyperamylasemia, significant pancreatic tissue necrosis, systemic inflammatory response, and lung injury $(26,28)$.

The targeted disruption of the ctsb gene and the complete absence of functional CTSB in the pancreas of these mice altered the course of acute pancreatitis in a number of ways. The most dramatic change in comparison to wild-type control animals, and also the most relevant in regard to the cathepsin-hypothesis of acute pancreatitis, was a significant reduction in the premature and intrapancreatic trypsinogen activation. In terms of substrate-defined trypsin activity, this reduction amounted to more than $90 \%$ over the course of 24 hours, when the greater pancreatic trypsinogen content of $\mathrm{CTSB}^{-/-}$animals is taken into account.

This prevention of premature trypsinogen activation was, however, incomplete. In terms of the presence of TAP in the pancreas, which indicates the total amount of trypsinogen that has been activated over time rather than the actual activity at a given point in time, the reduction of trypsinogen conversion that can be attributed to the absence of CTSB was approximately $50 \%$ and thus corresponded to the reduction of acinar cell necrosis. CTSB is therefore clearly not the only factor involved in premature intrapancreatic trypsinogen activation or cell injury and additional mechanisms such as trypsinogen autoactivation or a trypsinogen activation by other lysosomal cysteine proteinases, e.g., cathepsin $\mathrm{L}, \mathrm{H}$, and $\mathrm{S}$, must be considered as potential alternatives.

Although the reduction of trypsinogen activation corresponded to the decrease in acinar cell injury, the amount of cells undergoing apoptosis and, more surprisingly, the systemic inflammatory response did not. Neither the degree of leukocyte infiltration in the pancreas or lungs nor the histological degree of lung injury during pancreatitis was affected by the absence of CTSB. This may indicate that the presence or absence of CTSB is irrelevant for the development of systemic and inflammatory complications associated with acute pancreatitis. Alternatively, this observation might indi- cate that as long as pancreatic damage above a certain threshold level has occurred, the systemic complications of the disease evolve in a manner that is completely independent of the initial degree of trypsinogen activation or the extent of acinar cell injury.

In conclusion, we used CTSB-deficient mice to study whether CTSB is critically involved in premature zymogen activation and pancreatic damage in an experimental model of pancreatitis. Our results show that the presence or absence of CTSB is, indeed, an important factor that determines the degree of premature trypsinogen activation and the extent of acinar cell necrosis, but not the systemic inflammatory response associated with acute pancreatitis. To our knowledge, these data provide the first direct and conclusive experimental evidence for a role of cathepsin B in the onset of acute pancreatitis.

\section{Acknowledgments}

The authors thank P. Schmidt and A. Hafner (München, Germany) for evaluating histological sections; S. Dollwet (Freiburg, Germany), V. Krause (Magdeburg, Germany), N. Hartelt (Göttingen, Germany), and U. Breite (Münster, Germany) for excellent technical assistance; $P$. Saftig (Göttingen, Germany) for providing wild-type control mice; K. Rajewski (Köln, Germany) for the E-141 cell line; and E. Weber for a CTSB-specific antiserum. J. Deussing was supported by a fellowship grant of the Fonds der Chemischen Industrie. This study was supported by the DFG, the BMBF of Germany, the ZFKI Freiburg, and the IZKF Münster.

1. Chiari, H. 1896. Über die Selbstverdauung des menschlichen Pankreas. Zeitschrift für Heilkunde. 17:69-96.

2. Whitcomb, D.C. 1999. Early trypsinogen activation in acute pancreatitis. Gastroenterology. 116:770-772.

3. Lerch, M.M., et al. 1992. Acute necrotising pancreatitis in the opossum: earliest morphologic changes involve acinar cells. Gastroenterology. 103:205-213.

4. Whitcomb, D.C., et al. 1996. Hereditary pancreatitis is caused by a mutation in the cationic trypsinogen gene. Nat. Genet. 14:141-145.

5. Gudgeon, A.M., et al. 1990. Trypsinogen activation peptides assay in the early prediction of severity of acute pancreatitis. Lancet. 335:4-8.

6. Hofbauer, B., et al. 1998. Intra-acinar cell activation of trypsinogen during caerulein-induced pancreatitis in rats. Am. J. Physiol. 275:G352-G362.

7. Otani, T., Chepilko, S.M., Grendell, J.H., and Gorelick, F.S. 1998. Codistribution of TAP and the granule membrane protein GRAMP92 in rat caerulein-induced pancreatitis. Am. J. Physiol. 275:G999-G1009.

8. Steer, M.L., and Meldolesi, J. 1987. The cell biology of experimental pancreatitis. N. Engl. J. Med. 316:144-150.

9. Figarella, C., Miszczuk-Jamska, B., and Barrett, A. 1988. Possible lysosomal activation of pancreatic zymogens. Activation of both human trypsinogens by cathepsin B and spontaneous acid. Activation of human trypsinogen-1. Biol. Chem. Hoppe-Seyler. 369:293-298.

10. Saluja, A., et al. 1987. Subcellular redistribution of lysosomal enzymes during caerulein-induced pancreatitis. Am. J. Physiol. 253:G508-G516.

11. Watanabe, O., Baccino, F.M., Steer, M.L., and Meldolesi, J. 1984. Supramaximal caerulein stimulation and ultrastructure of rat pancreatic acinar cell: early morphological changes during development of experimental pancreatitis. Am. J. Physiol. 246:G457-G467.

12. Leach, S.D., Modlin, I.M., Scheele, G.A., and Gorelick, F.S. 1991. Intracellular activation of digestive zymogens in rat pancreatic acini. Stimulation by high does of cholecystokinin. J. Clin. Invest. 87:362-366.

13. Saluja, A.K., et al. 1997. Cerulein-induced in vitro activation of trypsinogen in rat pancreatic acini is mediated by cathepsin B. Gastroenterology. 113:304-310. 
14. Steer, M.L., and Saluja, A.K. 1993. Experimental acute pancreatitis: studies of the early events that lead to cell injury. In The pancreas: biol ogy, pathobiology, and disease. V.L.W. Go, et al., editors. Raven Press. New York, New York, USA. 489-500.

15. Deussing, J., et al. 1998. Cathepsin B and D are dispensable for major histocompatibility complex class II-mediated antigen presentation. Proc. Natl. Acad. Sci. USA. 95:4516-4521.

16. Qian, F., Frankfater, A., Chan, S.J., and Steiner, D.F. 1991. The structure of the mouse cathepsin B gene and its putative promoter. DNA Cell Biol. 10:159-168.

17. Thomas, K.R., and Capecchi, M.R. 1987. Site-directed mutagenesis by gene targeting in mouse embryo-derived stem cells. Cell. 51:503-512.

18. Hooper, M., Hardy, K., Handyside, A., Hunter, S., and Monk, M. 1987. HPRT-deficient (Lesch-Nyhan) mouse embryos derived from germline colonization by cultured cells. Nature. 326:292-295.

19. Kühn, R., Rajewski, K., and Müller, W. 1991. Generation and analysis of interleukin-4 deficient mice. Science. 254:707-710.

20. Köster, A., et al. 1993. Targeted disruption of the M(r) 46,000 mannose 6-phosphate receptor gene in mice results in misrouting of lysosomal proteins. EMBO J. 12:5219-5223.

21. Chirgwin, J.M., Przybla, A.E., MacDonald, R.J., and Rutter, W.J. 1979. Isolation of biologically active ribonucleic acid from sources enriched in ribonucleases. Biochemistry. 18:241-245.

22. Isbrandt, D., et al. 1994. Muccopolysaccharidosis VI (MaroteauxLamy syndrome): six unique arylsulfatase gene alleles causing vari able disease phenotypes. Am. J. Hum. Genet. 54:454-463.

23. Lyons, K., et al. 1989. Vgr-1, a mammalian gene related to Xenopus Vg1 , is a member of the transforming growth factor beta superfamily. Proc. Natl. Acad. Sci. USA. 86:4554-4558.

24. Owens, J.W., Gammon, K.L., and Stahl, P.D. 1975. Multiple forms of beta-glucuronidase in rat liver lysosomes and microsomes. Arch. Biochem. Biophys. 166:258-272.

25. Saftig, P., et al. 1995. Mice deficient for the lysosomal proteinase cathepsin D exhibit progressive atrophy for the intestinal mucosa and profound destruction of lymphoid cells. EMBO J. 14:3599-3608.

26. Barrett, A.J. and Kirschke, H. 1981. Cathepsin B, cathepsin H and cathepsin L. In Methods in enzymology. Vol. 80. L. Lorand, editor. Academic Press. New York, New York, USA. 535-561.

27. Niederau, C., Ferrell, L.D., and Grendell, J.H. 1985. Caeruleininduced acute necrotizing pancreatitis in mice: protective effects of proglumide, benzotript, and secretin. Gastroenterology. 88:1192-1204.

28. Grady, T., Saluja, A.K., Kaiser, A., and Steer, M.L. 1996. Edema and intrapancreatic trypsinogen activation precedes glutathione depletion during caerulein pancreatitis. Am. J. Physiol. 271:G20-G26.

29. Bhatia, M., et al. 1998. Role of substance P and the neurokinin 1 receptor in acute pancreatitis and pancreatitis-associated lung injury. Proc. Natl. Acad. Sci. USA. 95:4760-4765.

30. Williams, J.A., Korc, M., and Dormer, R.L. 1978. Action of secretagogues on a new preparation of functionally intact, isolated pancreatic acini. Am. J. Physiol. 235:E517-E524.

31. Bradford, M.M. 1976. A rapid and sensitive method for the quanti- tation of microgram quantities of protein utilizing the principle of protein-dye binding. Anal. Biochem. 72:248-254.

32. Gavrieli, Y., Shearman, Y., and Ben-Sasson, S.A. 1992. Identification of programmed cell death in situ via specific labeling of nuclear DNA fragmentation. J. Cell Biol. 119:493-501.

33. Lerch, M.M., Saluja, A.K., Rünzi, M., Dawra, R., and Steer, M.L. 1995. Luminal endocytosis and intracellular targeting by acinar cells during early biliary pancreatitis in the opossum. J. Clin. Invest. 95:2222-2231

34. Jensen, R.T., Lemp, G.F., and Gardner, J.D. 1980. Interaction of cholecystokinin with specific membrane receptors on pancreatic acinar cells. Proc. Natl. Acad. Sci. USA. 77:2079-2083.

35. Balthazar, E.J., Robinson, D.L., Megibow, A.J., and Ranson, J.H. 1990. Acute pancreatitis: value of CT in establishing prognosis. Radiology. 174:331-336

36. Lerch, M.M., et al. 1997. Dissociation and reassembly of adherens junctions during experimental acute pancreatitis. Gastroenterology. 113:1355-1366

37. Gukovskaya, A.S., et al. 1996. Mechanisms of cell death after pancreatic duct obstruction in the opossum and the rat. Gastroenterology. 110:875-884

38. Kaiser, A.M., Saluja, A.K., Sengupta, A., Saluja, M., and Steer, M.L. 1995. Relationship between severity, necrosis, and apoptosis in five models of experimental acute pancreatitis. Am. J. Physiol. 269:C1295-C1304

39. Roberts, L.R., et al. 1997. Cathepsin B contributes to bile salt-induced apoptosis of rat hepatocytes. Gastroenterology. 113:1714-1726.

40. Leser, H.G., et al. 1991. Elevation of serum interleukin- 6 concentration precedes acute-phase response and reflects severity in acute pancreatitis. Gastroenterology. 101:782-785.

41. Lerch, M.M., and Adler, G. 1994. Pathophysiology of acute pancreatitis. Digestive Surgery. 11:186-192.

42. Tooze, J., Hollinshead, M., Hensel, G., Kern, H.F., and Hoflack, B. 1991. Regulated secretion of mature cathepsin B from rat exocrine pancreatic cells. Eur. J. Cell Biol. 56:187-200.

43. Lerch, M.M., Saluja, A.K., Dawra, R., Saluja, M., and Steer, M.L. 1993. The effect of chloroquine administration on two experimental models of acute pancreatitis. Gastroenterology. 104:1768-1779.

44. Gorelick, F.S., Modlin, I.M., Leach, S.D., Carangelo, R., and Katz, M. 1992. Intracellular proteolysis of pancreatic zymogens. Yale J. Biol. Med. 65:407-420.

45. Klonowski-Stumpe, H., et al. 1998. Inhibition of cathepsin B does not affect the intracellular activation of trypsinogen by cerulein hyperstimulation in isolated rat pancreatic acinar cells. Pancreas. 16:96-101.

46. Lerch, M.M., and Adler, G. 1994. Experimental animal models of acute pancreatitis. Int. J. Pancreatol. 15:159-170.

47. Ferrara, M., et al. 1990. Gene structure of mouse cathepsin B. FEBS Lett. 273:195-199.

48. Rhaissi, H., Béchet, D., and Ferrara, M. 1993. Multiple leader sequences for mouse cathepsin B mRNA. Biochemistry. 75:899-904. 